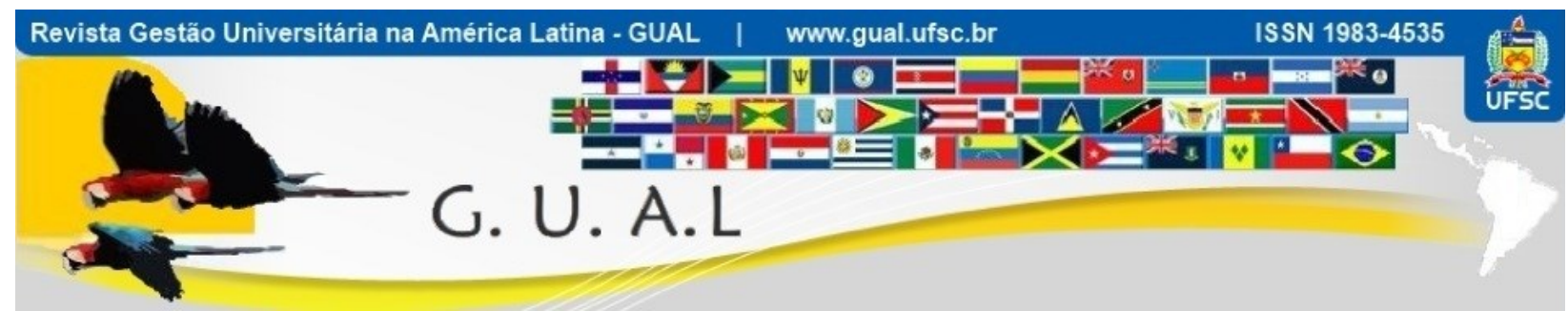

DOI: http://dx.doi.org/10.5007/1983-4535.2013v6n2p55

\title{
RESISTÊNCIA À MUDANÇA NA EDUCAÇÃO SUPERIOR: DESIGN E OPERACIONALIZAÇÃO DE UM INSTRUMENTO DE MEDIDA PARA O MEES
}

\section{RESISTANCE TO CHANGE IN HIGHER EDUCATION: DESIGN AND IMPLEMENTATION OF A MEASURING INSTRUMENT FOR THE MEES}

\author{
Angela Cristina Corrêa, Doutora \\ Universidade Federal de Santa Catarina - UFSC \\ angelaccorrea@gmail.com \\ Silvana Ligia Vincenzi Bortolotti, Doutora \\ Universidade Tecnológica Federal do Paraná - UTFPR \\ sligie@globo.com \\ Afonso Farias de Sousa Júnior, Doutor \\ Universidade da Força Aérea - UNIFA \\ bvfire@gmail.com \\ Dalton Francisco de Andrade, Doutor \\ Universidade Federal de Santa Catarina - UFSC \\ dandrade@inf.ufsc.br \\ Fernando de Jesus Moreira Junior, Doutor \\ Universidade Federal de Santa Maria - UFSM \\ fmjunior@smail.ufsm.br \\ Raquel Dalvit Flores, Mestranda \\ Universidade Federal de Santa Maria - UFSM \\ raqueldalvit@hotmail.com
}

Recebido em 29/julho/2012

Aprovado em 29/março/2013

Sistema de Avaliação: Double Blind Review 


\title{
RESUMO
}

A autonomia científica e tecnológica de um país pressupõe que o mesmo tenha uma educação de qualidade. O objetivo deste artigo é apresentar um instrumento de medida de avaliação da resistência à mudança na administração da educação superior. Propõe-se um desenho e operacionalização para o projeto Mapa Estratégico da Educação Superior (MEES) fundamentado em um sistema de gestão integrado. O MEES se constitui em uma proposta metodológica para a operacionalização do Plano de Desenvolvimento Institucional (PDI) em Instituições de Ensino Superior (IES). O instrumento de medida de avaliação da resistência à mudança na administração da educação superior (RAMES) se fundamenta na seguinte base conceitual: teoria de resposta ao ítem (TRI), teoria psicometrica e no estudo sobre gestão da mudança. A RAMES compõe-se de cinco etapas: 1. elaboração do instrumento de coleta de dados; 2. definição do tamanho amostra representativa da comunidade acadêmica das IES; 3 . coleta de dados - conjunto de itens; 4. estimação dos parâmetros dos itens; 5 . definição da escala de medida A RAMES tem como expectativa de resultados contribuir para a eficácia na implementação do MEES. Visa também identificar os principais indicadores de resistência a mudança na administração da educação superior.

Palavras-chave: Resistência a mudança. Teoria de resposta ao ítem. Educação superior. Estratégia. Teoria psicométrica.

\begin{abstract}
The scientific and technological autonomy of a country requires it to have a exceptional education. The aim of this paper is to present an assessment instrument to measure the resistance to change in the administration of higher education. We propose a design and operation for the project Strategic Map of Higher Education (MEES) based on an integrated management system. The MEES constitutes a methodological proposal for the operationalization of the Institutional Development Plan (IDP) in Higher Education Institutions (HEI). A measuring instrument for assessing the resistance to change in the administration of higher education (RAMES) is based on the following conceptual basis: item response theory (IRT) psychometric theory and the study of change management. The Rames consists of five steps: 1 . development of the instrument for data collection 2. definition of the size of the sample of the academic community inside the HEIs, 3. Data collection - set of items 4. estimate the parameters of items: 5 . definition of the measurement scale. The Rames is expected to contribute to the effectiveness in implementing the MEES. It also aims to identify key indicators of resistance to change in the administration of higher education.
\end{abstract}

Keywords: Resistance to change. Item response theory. Higher education. Strategy. Psychometric theory.

APOIO: Este artigo foi apoiado e financiado pelo Edital Pró-administração da CAPES. 


\section{INTRODUÇÃO}

A abordagem clássica à educação ainda hegêmonica, muito contribuiu para as inovações e revoluções no campo científico, tecnológico, artístico e cultural. No entanto, questiona-se a sua efetividade para a resolução de problemas cruciais da humanidade, tais como o superaquecimento da terra, a poluição, a fome, a desigualdade de renda, analfabetismo, conflitos entre nações, terrorismo e catástrofes ambientais.

O enfoque mecanicista da abordagem clássica está sendo substituído gradativamente por uma percepção sistêmica. Não há mais espaço para a gestão que priorize metas e resultados em detrimento da dimensão humana e de mapeamento de processos. Para criar a consciência desta transformação na maneira como está sendo conduzido o pensamento da humanidade é necessário uma preparação, sensibilização para as mudanças advindas deste processo.

Segundo Meyer (1997), embora as universidades sejam o lócus onde são preparados futuras gerações de profissionais e cidadãos e onde são feitas reflexões acerca do mundo e da vida em sociedade e discutidas alternativas para um mundo mais justo e melhor, paradoxalmente é nestas mesmas universidades que se encontram as maiores resistências às mudanças e transformações exigidas pelo ambiente.

O objetivo deste artigo é apresentar um instrumento de medida de avaliação da resistência à mudança na administração da educação superior. Propõe-se um desenho e operacionalização para o projeto Mapa Estratégico da Educação Superior(MEES) fundamentado em um sistema de gestão integrado. O MEES constitui-se em uma proposta metodológica para a implementação do Plano de Desenvolvimento Institucional (PDI) em Instituições de Educação Superior Brasileiras (IES). O mapa estratégico se constitui na representação gráfica do sistema de gestão integrado.

$\mathrm{O}$ instrumento de medida de avaliação da resistência à mudança na administraçaõ da educação superior se fundamenta na seguinte base conceitual: teoria de resposta ao ítem (TRI), na teoria psicometrica e no estudo sobre gestão da mudança.

\section{GESTÃO DA MUDANÇA: CONCEITOS, RESISTÊNCIA E ATITUDES}

$\mathrm{Na}$ área de mudança organizacional a literatura é vasta, uma vez que impacta diretamente nos resultados a alcançar e na vida organizacional, pois se trata de um campo emergente na vida organizacional. 


\subsection{Conceitos}

A concepção, o tipo e processo de gestão da mudança que norteiam este estudo têm um enfoque multidimensional, ou seja, considera as relações de poder, cultura, princípios, cadeia e a rede de valor, dentre entre outros aspectos.

Nesta abordagem, o conceito de mudança fundamenta-se precipuamente nos seguintes autores: Wood Jr. (2004), Robbins (2002), Neiva (2004), Valle-Lima (2003), Nadler et al. (1995), Porras e Robertson (1992).

A literatura aponta vários tipos de mudança organizacional, onde se destacam os seguintes autores: Weick \& Quinn (1999), Nadler, Shaw, Walton e cols. (1995), Porras \& Robertson (1992), Silva (1999).

A mudança que está em estudo se enquadra no tipo proposto por Porras e Roberson (1992), denominada de segunda ordem, a qual se caracteriza como sendo multidimensional, multínivel, descontínua e envolve quebra de paradigmas organizacionais.

O processo de gestão da mudança compreende um conjunto de ações, reações e interações entre as várias partes interessadas, na medida em que procuram alterar a organização levando-a de um estado presente tendo em vista um estado futuro (PETTIGREW, 1989). Para Alperstedt (2000), a análise do processo compreende o "como" da mudança e consiste na forma e nas estratégias pelas quais ela se desenvolve e é conduzida.

\subsection{Resistência}

Algumas pesquisas realizadas, como a de Maurer, (1996) e Pascale et al. (1997), revelaram pelo menos metade de todas as mudanças propostas tendem ao fracasso e a grande maioria das mudanças não conseguem atingir os objetivos pretendidos (GILMORE et al., 1997). As causas dos resultados do fracasso da mudança são vários como, por exemplo, o fato de que os gestores não conseguiram entender inteiramente o que é preciso para conduzir a implantação de uma mudança (SELF; SCHRAEDER, 2008). Porém, há também outro fator que pode influenciar neste fracasso que é a resistência à mudança (DEL VAL, 2003) e para alguns a resistência é considerada a ameaça mais significante para o sucesso de uma mudança (MAURER, 1996; GEISLER, 2001).

Assim, um planejamento adequado da implementação que leve em consideração as causas de resistência (WADELL; SOHAL, 1998, O’CONNOR, 1993. JUDSON, 1980) 
podem ajudar a mitigar a probabilidade de fracasso de mudança e também ajudar a evitar indesejáveis conseqüências (SELF; SCHRAEDER, 2008).

A resistência resulta de diferenças, tais como: idéias, motivos, planos, prioridades. Se os gestores de mudança aceitarem que há diferentes pontos de vista, eles podem trabalhar para integrar estas diferenças e, consequentemente, minimizar as resistências. Entender as causas da resistência, criar resoluções e tentar minimizá-la, constitui um passo vital (O’CONNOR, 1993).

Resistência é tida como "força que retarda ou para um movimento" (MAURER, 1996, pag 23), já Watson (1971, p.745) define a resistência como "todas as forças que contribuem para a estabilidade nos sistemas da personalidade ou social". Destaca que a resistência à mudança tem sido por vezes, mal interpretada como simples inércia na natureza humana, mas que na verdade, quase todos estão ansiosos por alguma espécie de mudança de vida, ou situação, que gostariam de melhorar; como por exemplo: mais dinheiro e mais liberdade para satisfazerem seus desejos. Segundo o mesmo autor, o motivo pelo qual as pessoas e as organizações não mudam deve-se às forças naturais em direção à inovação que as impedem de mudar, ou são bloqueados por forças antagônicas.

No contexto organizacional, a resistência é uma expressão que normalmente surge como uma resposta ou uma reação à mudança (BLOCK, 1989, p. 199). Esta expressão normalmente é observada pelo gerente como quaisquer ações percebidas do empregado na tentativa de parar, retardar, ou alterar a mudança (BEMMELS; RESHEF, 1991). Kotter (1995) sugere que a resistência é um obstáculo na estrutura da organização que impede a mudança. Ou também, é qualquer comportamento percebido dos membros da organização que parecem relutantes em aceitar ou ajudar a implementar uma mudança organizacional (COGHLAN, 1993).

As diversas abordagens sugerem que as resistências às mudanças nas organizações têm suas origens centradas nas características pessoais dos indivíduos, como, percepções, personalidades e necessidades e, também no contexto organizacional. Chiavenato (2005) salienta que a resistência à mudança pode ser consequência de aspectos: lógicos que envolvem objeções racionais e lógicas; psicológicos englobam atitudes emocionais e psicológicas e sociológicos abarcam interesses de grupos e fatores sociológicos.

Em relação as características pessoais os indivíduos podem resistir por sentirem medo (KOTTER \& SCHLESINGER, 1979; ROBBINS, 2002; TAYLOR, 1988). Este medo pode 
vir do sentimento por: a) um interesse pessoal, isto é, de perder algo de valor (KOTTER; SCHLESINGER, 1979; TAYLOR, 1988); b) por uma diminuição na segurança profissional e de benefícios econômicos, ou seja, de redução dos rendimentos (ROBBINS, 2002; JUDSON, 1966; TAYLOR, 1988) c) medo do desconhecido, (KARIM E KATHAWALA, 2005; ROBBINS, 2002; VISAGIE E BOTHA, 1998), d) de perder o controle das coisas que conquistou (CONNER, 1992; OREG, 2003) ou incertezas, porque eles estão preocupados com seu proprio fracasso (MINK, 1992).

Um estudo, envolvendo variáveis psicológicas na investigação do processo de mudança organizacional, foi conduzido por Judge et al. (1999) para verificar se o lidar com a mudança está relacionado com a predisposição psicológica dos indivíduos que a experimentam. Neste estudo eles verificaram que a gestão para lidar com a mudança organizacional é influenciada por sete características da personalidade e dentre elas, a abertura à experiência. Indivíduos com alta abertura à experiência estão mais dispostos em tentar coisas novas (NIKOLAOU et al., 2007). Já indivíduos com níveis mais baixos de abertura à experiência, são mais prováveis que sejam menos interessados em tentar coisas novas.

Há várias outras causas de resistência à mudança tanto relacionadas com variáveis individuais como de contexto organizacionais e também de resultados esperados do trabalho. Dentre as outras causas individuais de resistência à mudança existentes podemos citar a abertura à experiência (Judge et al.,1999), etnocentrismo cultural (ZALTMAN e DUNCAN, 1979), dogmatismo (OREG, 2006; BARTUNEK, LACEY, WOOD, 1992; BARTUNEK e MOCH, 1987; LAU e WOODMAN, 1995), resiliência psicológica (OREG, 2006). Já dentre variáveis de contexto organizacionais têm-se: participação (OREG, 2006; WANBERG e BANAS, 2000), poder e prestígio (OREG, 2006; HIETAPELTO, 2002; STEWART, MANZ, 1997), comprometimento organizacional (OREG, 2006, canais de comunicação e informação (WANBERG e BANAS, 2000; Miller et al., 1994; WANBERG e BANAS, 2000; COCH e FRENCH, 1948; KOTTER e SCHLESINGER, 1979), conflitos internos e processo decisório (SCHEIN,1982), influência social (OREG, 2006), recompensa intrínseca (OREG, 2006; HACKMAN e OLDHAM, 1980; TICHY, 1983) e confiança à gerência (OREG, 2006; KOTTER, 1995; ZANDER, 1950; STANLEY, MEYER e TOPOLNYTSKI 2005).

A manifestação da resistência à mudança se dá de diversas formas, umas mais aparentes ou mais sutis do que outras, mas todas provocam efeitos negativos para a 
organização, e depende da personalidade do indivíduo, da natureza da mudança, das atitudes destes para com tal mudança, e das forças que derivam do grupo e da organização em seus contextos (JUDSON, 1966; MENDES, 2001).

Hultman (1995), Judson (1980), Moscovici (1994), Mendes (2001) e Giangreco (2002), apresentam diversas formas de manifestação da resistência à mudança, dentre elas ressaltam: Resistência Ativa, Resistência Passiva, indiferença e aceitação.

\subsection{Atitude}

Ao se falar em mudanças, se fala de problemas que têm haver com as atitudes das pessoas frente às mudanças.

O conceito de atitude é um dos mais estudados e antigos da Psicologia Social. Este conceito faz uma ligação entre as disposições individuais e idéias socialmente partilhadas e, depois, as suas formas de avaliação (escalas de atitudes) deram identidade à Psicologia Social (VALA; MONTEIRO, 2006).

As atitudes dos indivíduos frente à mudança organizacional adquirem caráter fundamental quando se assinala seu papel no sucesso dos processos de mudança organizacional (NEIVA, 2004).

O conceito adotado de resistência neste trabalho é o de Piderit (2000), que a analisa sob o conceito de atitudes da Psicologia Social. Para Piderit (2000), resistência à mudança é definida como uma atitude ambivalente na resposta inicial do empregado para com a mudança. Desta forma, a mesma autora considera resistência à mudança como sendo uma atitude, incluindo componentes afetivos, comportamentais e cognitivos. Os três componentes não são independentes um do outro, e o que as pessoas sentem sobre uma mudança freqüentemente corresponderá com o que elas pensam sobre a própria e com respeito a suas intenções comportamentais (OREG, 2006).

\section{O CONCEITO DE MEDIDA SOB A OTICA DA TEORIA PSICOMÉTRICA}

Uma das três grandes linhas da teoria da medida por teoria é, a teoria das escalas e testes (a psicometria). Esta linha aborda a medida de construtos ou de traços latentes por meio de manifestações observáveis, que seriam a representação da variável hipotética. Os parâmetros são a legitimidade de tal representação (validade) e a análise de itens em termos 
de dificuldade e discriminação, determinados através da Teoria da Resposta ao Item (PASQUALI, 1996, 2003).

O traço latente é representado por uma variedade de expressões, tais como: variável hipotética, fator, traço latente, conceito, habilidade, aptidão, atitude, componente cognitivo e traço da personalidade (PASQUALI, 2003).

O objeto de estudo ou de interesse da psicometria é o traço latente. O traço latente em estudo é a resistencia à mudança na administraçao da educação superior e seus elementos constituintes: ensino, pesquisa, extensão/assistência e a gestão propriamente dita.

\subsection{Procedimentos para elaborar um conjunto de itens de avaliação}

O modelo adotado para a modelagem do instrumento de avaliação da resistência na administração da educação superior obedece aos procedimentos propostos por Pasquali (1998), que fornece uma estrutura prática de se obter a validade do instrumento de medida. O autor estabelece uma esquematização para a construção de um conjunto de itens, a qual compreende três procedimentos: teóricos, experimentais e analíticos.

\section{CONTRIBUIÇÕES DA TRI PARA AVALIAR A RESISTÊNCIA A MUDANÇA NAS ORGANIZAÇÕES}

Como a resistência à mudança é um traço latente, ou seja, é uma característica que não pode ser medida diretamente, para avalia-la é necessário um conjunto de variáveis secundárias que estejam relacionadas com ela. Existem vários modelos para avaliar traços latentes.

A TRI vem ganhando cada vez mais espaço, sendo aplicado em diversas áreas do conhecimento, mas com mais freqüência nas avaliações educacionais, devido as grandes vantagens oferecidas em relação à Teoria Clássica dos Testes. Uma relação de trabalhos desenvolvidos com a TRI no Brasil até o ano de 2009 pode ser encontrada em Moreira Junior (2010).

A TRI fornece modelos matemáticos para os traços latentes, propondo formas de representar a relação entre a probabilidade de um indivíduo dar uma certa resposta a um item, seu traço latente e características (parâmetros) dos itens, na área de conhecimento em estudo (ANDRADE; TAVARES E VALLE, 2000).

A TRI relaciona-se com a estimação de parâmetros dos itens e os parâmetros dos indivíduos em uma medida. Por exemplo, considere o traço latente resistência à mudança, uma análise feita por meio da TRI, estima o nível de resistência do respondente (isto é, um 
parâmetro do indivíduo) e também como os itens na medida discriminam entre indivíduos com níveis diferentes de resistência (isto é, um parâmetro do item) (Bortolotti, 2010).

A TRI possibilita que os parâmetros dos itens sejam invariantes sobre os respondentes e os traços latentes dos respondentes sejam invariantes sobre os itens, exceto pela escolha de origem e escala (VAN DER LINDEN E HAMBLETON, 1997). Ademais, uma escala criada com base na TRI tem a vantagem de colocar tanto os itens como os respondentes numa mesma escala de medida, pois com a aplicação de um dos modelos da TRI ao conjunto de respostas dadas a um instrumento de medida obtido dos respondentes estimam-se os parâmetros dos itens e dos respondentes numa única escala.

Os modelos da TRI dependem do tipo de item e do tipo de processo de resposta. Eles podem ser acumulativos ou não acumulativos. Os modelos acumulativos da TRI são modelos em que a probabilidade de um indivíduo dar ou escolher uma resposta correta ao item aumenta com o aumento do seu traço latente, isto é, níveis maiores de traço latente conduzem a valores mais altos de probabilidade de resposta correta, apresentando um comportamento monotônico na CCI.

O modelo de desdobramento graduado generalizado (GGUM), é um modelo de Teoria da Resposta ao Item, unidimensional, desenvolvido para analisar tanto respostas binárias quanto graduadas. O GGUM tem como aplicação típica, situações de medidas onde os entrevistados são solicitados a indicarem seu nível de concordância, a um conjunto de itens que se situam numa escala bipolar. Ou seja, que variam o seu conteúdo do negativo para o positivo, passando pelo neutro. Este modelo é adequado para analisar dados de atitude, em especial, dados referentes a resistência à mudança.

No GGUM tem-se que quando um indivíduo é solicitado para expressar a sua opinião de aceitação, em uma declaração de atitude, o indivíduo tende a concordar com o item à medida que ele é localizado próximo de sua posição em uma escala do traço latente (ROBERTS; DONOGHUE; LAUGHLIN, 2000).

Os parâmetros dos itens serão obtidos por meio do software GGUM2004 (ROBERTS et al, 2004).

\section{A ADMINISTRAÇÃO DA EDUCAÇÃO SUPERIOR NO BRASIL}

O documento do Fórum Nacional da Educação Superior (FNES), que contribuiu para a elaboração do Plano Nacional da Educação (PNE 2011-2020), destaca três eixos que merecem 
especial atenção para a construção das políticas públicas no Brasil: 1) democratização do acesso e flexibilização de modelos de formação; 2) elevação da qualidade e avaliação; 3) compromisso social e inovação (Ronca et al., 2009)

Os autores supracitados destacam que como democratização do acesso e flexibilização dos modelos de formação acadêmica, o Fórum entende que fazer do acesso à ES um direito implica, pois, na implantação de políticas de inclusão e de equidade que promovam mudanças na identidade e missão das instituições. Estas devem transformar-se tendo como elemento central a pertinência e a responsabilidade social, de acordo com os recursos disponíveis. Para tanto, deve haver opções de acesso baseadas em diferentes habilidades e, ainda, em diferentes opções de cursos, currículos e trajetórias institucionais que, em atenção a diversos grupos e setores sociais, como mulheres, grupos de terceira idade, movimentos sociais - promovam experiências de inclusão, multidiversidade cultural e educação para toda a vida.

\section{O SISTEMA NACIONAL DE AVALIAÇÃO DA EDUCAÇÃO SUPERIOR (SINAES) E O PLANO DE DESENVOLVIMENTO INSTITUCIONAL (PDI)}

O Ministério da Educação instituiu o Sistema Nacional de Avaliação da Educação Superior (SINAES) - lei 10.861, de 14 de Abril de 2004, com o propósito de realizar uma avaliação diagnóstica, formativa e regulatória das IES (BRASIL, 2004).

O SINAES dispõe de alguns instrumentos que dão suporte ao gerenciamento da educação superior nas suas dimensões pedagógica, avaliativa e estratégica. O Projeto Pedagógico Institucional (PPI) apresenta a concepção filosófica e pedagógica de suas práticas acadêmicas; o sistema de avaliação composto de vários subsistemas: auto-avaliação institucional e avaliação externa, avaliação de cursos de graduação e do desempenho de estudantes (ENADE); E, articulado a todos estes instrumentos o Plano de Desenvolvimento Institucional (PDI), o qual se constitui em um plano de acompanhamento da concretização de sua missão, objetivos e metas (INEP, 2009).

O PDI deve considerar a missão, os objetivos e as metas da instituição, bem como as propostas de desenvolvimento das suas atividades, definindo claramente os procedimentos relativos à qualificação do corpo docente, inclusive quanto a eventuais substituições, assim como o regime de trabalho, o plano de carreira, a titulação, a experiência profissional no magistério superior e a experiência profissional não acadêmica, levando em conta as condições de formação em pós-graduação de docentes na região, o projeto pedagógico dos cursos e as outras atribuições acadêmicas dos docentes (INEP, 2009). 
O PDI é elaborado para um período determinado e configura-se como um instrumento de gestão flexível, que se pauta em objetivos e metas e sua elaboração deve ser de caráter coletivo. Articula-se ao PPI e seus referenciais devem levar em consideração os resultados da avaliação institucional (MEC, 2006).

Corrêa (2007) e Corrêa et al. (2008) relatam que as suas experiências como avaliadores de cursos de graduação, demonstram que muitos dos instrumentos do SINAES estão cumprindo uma função meramente formal. Não estão sendo construídos coletivamente com a participação efetiva da comunidade acadêmica, conforme preconizam as diretrizes do SINAES.

$\mathrm{Na}$ fase exploratória deste estudo constatou-se por meio da realização de um benchmarking dos PPIs de algumas IES, que uma parcela significativa foi construída desarticuladamente dos demais instrumentos do SINAES. Verificou-se também que um problema comum no processo de elaboração do PDI e do PPI e nos instrumentos de avaliação, é que os mesmos não são acompanhados de um plano de implementação operacional.

Constata-se a necessidade de uma otimização dos instrumentos de planejamento e avaliação do MEC, com o propósito de: evitar duplicidade de informações e permitir a convergência de esforços e a sua implementação de maneira dinâmica, sistêmica e integrada. O sistema de gestão integrado apresentado na seção seguinte se constitui em uma proposta metodológica para a operacionalização do PDI.

\section{O MAPA ESTRATÉGICO DA EDUCAÇÃO SUPERIOR (MEES) FUNDAMENTADO EM UM SISTEMA DE GESTÃO INTEGRADO: UMA PROPOSTA METODOLÓGICA PARA A OPERACIONALIZAÇÃO DO PDI}

\subsection{Concepção do sistema}

O mapa estratégico é a representação visual de um sistema de gestão integrado para as IES, o qual se constitui em uma proposta metodológica para operacionalização do PDI fundamentado na abordagem quântica (cf. Figura 1). 


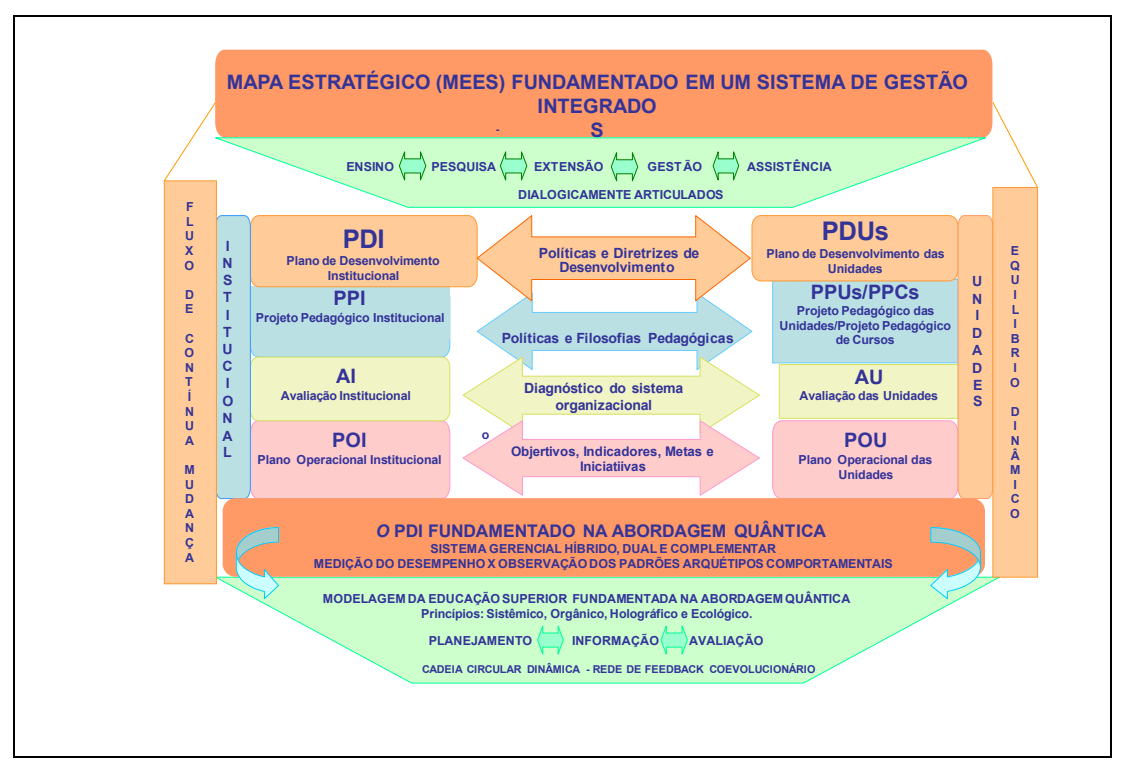

Figura 1 Mapa Estratégico da Educação Superior Fundamentado em um Sistema de Gestão Integrado: Uma proposta metodológica para a operacionalização do PDI fundamentado na Abordagem Quântica.

Fonte: Corrêa $(2007,2008)$.

Incorpora aos instrumentos de planejamento e avaliação do Sistema Nacional de Avaliação da Educação Superior, uma concepção alternativa à educação superior. O PDI é construído coletivamente a partir das bases da instituição, por meio dos seguintes instrumentos: Plano de Desenvolvimento da Unidade (PDU) e seus subsistemas: Projeto Pedagógico da Unidade (PPU), Avaliação da Unidade (AU) e Plano Operacional da Unidade (POI).

A avaliação procede a um diagnóstico organizacional da educação superior à luz do SINAES (auto-avaliação, avaliação externa, ENADE e avaliação de cursos de graduação) e da CAPES (ensino de pós-graduação). E o plano estratégico, com base no PPI, AI e pensamento estratégico institucional (visão, missão, princípios e valores) estabelece objetivos, indicadores, metas e iniciativas a curto, médio e longo prazo.

A abordagem quântica à educação superior fundamenta-se em quatro princípios: sistêmico, orgânico, holográfico e ecológico. O princípio sistêmico concebe o sistema de gestão como interativo, o qual visa integrar as políticas, diretrizes e estratégias institucionais com as setoriais. O princípio holográfico visualiza a instituição como um holograma, os Planos de Desenvolvimento das Unidades (PDUs) estão contidos no PDI e vice-versa, simultaneamente. O princípio ecológico extrapola as questões ambientais. Os propósitos e valores do sistema universitário definem a ecologia do ambiente. $\mathrm{O}$ princípio orgânico elege 
como requisitos de um sistema sob a ótica quântica a flexibilidade e a adaptabilidade das estratégias da educação superior às constantes mutações do ambiente.

O Mapa Estratégico para a Educação Superior (MEES) é a ferramenta de representação visual da dimensão estratégica do PDI fundamentado na abordagem quântica. Fundamenta-se no Mapa Estratégico do Balanced Scorecard como um Sistema Complexo Adaptativo - 'BSC como um SCA', o qual consiste numa modelagem adaptativa ao modelo proposto por seus idealizadores: Robert Kaplan e David Norton (Corrêa, 2005, 1a, 1b).

O MEES deve ser utilizado como uma ferramenta de descrição, aprendizado e reflexão nas reuniões e workshops de comissões e grupos de trabalho, e consequentemente contribui para criar a consciência estratégica. O mapa estratégico da educação superior visa apresentar a sociedade os objetivos, indicadores, metas e iniciativas estratégicas com transparência e continuidade. A consciência estratégica será construída coletivamente.

\subsection{Plano de implementação}

O Plano de implementação do Sistema de Gestão Integrado (PDI/PDUs) compõe-se basicamente de três fases: institucionalização, implementação do Primeiro e Segundo Ciclos.

\subsubsection{Fase 1: Institucionalização}

O comprometimento da administração central da Instituição e das Unidades de Ensino é fundamental para a eficácia do sistema de gestão integrado PDI/PDUs. O Sistema de Gestão Integrado PDI/PDUs deverá ser institucionalizado por meio de Resolução normativa. Deverá prever e nomear por meio de portarias os membros para a constituição da Comissão do Plano de Desenvolvimento Institucional (CPDI) e as Comissões dos Planos de Desenvolvimento das Unidades (CPDUs).

\subsubsection{Fase 2: Implementação do $1^{\circ}$ Ciclo - Identidade da IES}

No $1^{\circ}$ Ciclo nenhuma ação de mobilização para a mudança é realizada com o intuito de resgatar a identidade, o processo de auto-referência das instituições. Partindo-se do pressuposto que uma instituição se constitui de pessoas que constituem a sua identidade, o $1^{\circ}$ Ciclo têm a finalidade de gerar o autoconhecimento institucional e das unidades integrantes. 


\subsubsection{Fase 3: Implementação do $2^{\circ}$ Ciclo}

O segundo ciclo tem inicio com a mobilização para a mudança por meio da incorporação da abordagem quântica na IES, com o propósito de produzir mudanças, gerar interações pró-ativas e dinâmicas e transformar os padrões arquétipos comportamentais reativos em pró-ativos.

As demais etapas consistem numa reflexão e revisão dos processos desenvolvidos no $1^{\circ}$ Ciclo. Ao final do $2^{\circ}$ Ciclo têm-se consolidado o PDI, como um sistema de gestão integrado, dual e complementar, de medição de desempenho (objetivos/metas e resultados) e observação dos padrões arquétipos comportamentais (reflexão sobre a inteligência emocional, motivacional e cognitiva) da instituição de ensino superior.

\subsubsection{Avaliação do Feedback estratégico}

Ao término de cada ciclo de implementação do sistema de gestão integrado (PDI/PDUs) se procede a uma apreciação crítica sobre a gestão estratégica da educação superior. Nesta etapa que se pretende utilizar o instrumento de medida para avaliar a resistência a mudança.

\section{DELINEAMENTO METODOLÓGICO DO ESTUDO}

A Figura 2 apresenta o delineamento metodológico deste estudo. Classificando a pesquisa na sua concepção filosófica, enquadra-se na abordagem quântica sob os fundamentos de algumas teorias. A teoria inserida em uma determinada abordagem do conhecimento reflete um paradigma científico, ou seja, um modelo de mundo, percepções e valores inerentes a um contexto histórico e social.

Adotar-se-á métodos diferenciados em cada fase de pesquisa, ressaltando que eles terão a função de balizar este estudo de forma que o mesmo seja desenvolvido de maneira sistemática e lógica, seguindo os preceitos da pesquisa científica.

As metodologias das correntes filosóficas que nos permitem refletir sobre as questões econômicas, sociais e culturais da sociedade, levam as organizações a colocar a reflexão, a imaginação no mesmo patamar de importância que as técnicas e métodos de avaliação e gestão organizacional.

Segundo Morgan (1996, p.271), ao estabelecer-se relação dialéticas, não se está abordando os processos lineares nos quais A é causa de B. Ao contrário, procuram-se 
oposições em que um lado do fenômeno tende a gerar a existência de outro. O hábito de "pensar linearmente" tende a bloquear a capacidade de pensar dialeticamente.

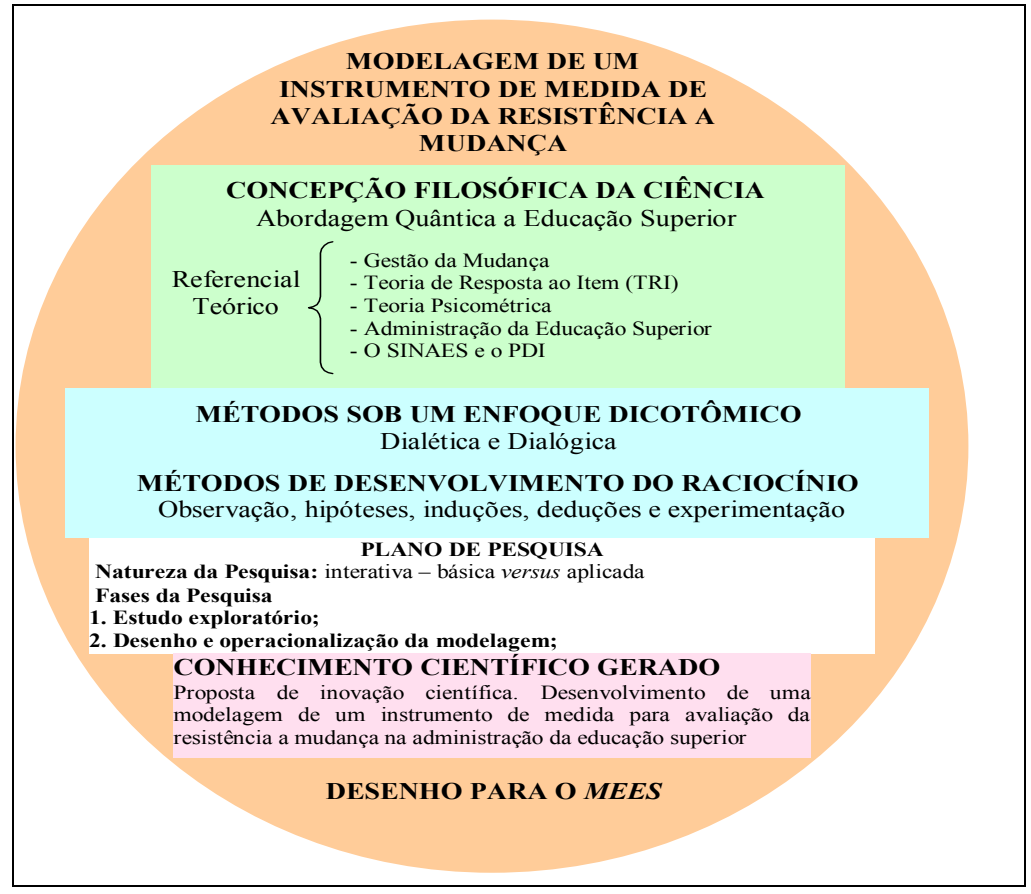

Figura 2 Delineamento Metodológico de um Instrumento de Medida de Avaliação da Resistência a Mudança na Administração da Educação Superior: desenho para o MEES.

Segundo Morin (1998), no Pensamento dialógico, duas lógicas, dois princípios, estão unidos sem que a dualidade se perca nessa unidade: daí vem à idéia de "unidualidade" para certos casos; desse modo, o homem é um ser unidual, totalmente biológico e totalmente cultural a um só tempo).

Este estudo quanto a sua natureza classifica-se como interativo: básica versus aplicada. Caracteriza-se como básica quando desenvolve uma modelagem conceitual de um instrumento de medida de avaliação da resistência a mudança para a gestão da educação superior; E como aplicada quando constroi um desenho para um sistema de gestão integrado para a operacionalização do PDI em IES. O estudo constituiu-se de duas etapas: a pesquisa exploratória e o desenvolvimento da modelagem. A pesquisa exploratória consiste na revisão bibliográfica e estado da arte sobre a fundamentação teórica que norteia este estudo.

A modelagem constitui-se basicamente em uma aplicação da tese de Bortolotti (2010) para a educação superior por meio de um desenho para um sistema de gestão integrado para a operacionalização do PDI proposto por Corrêa (2007) e Corrêa et al. (2008). Este estudo faz parte das missões de estudo do projeto "Mapa estratégico da Educação Superior (MEES) 
fundamentado em um Sistema de Gestão Integrado: uma proposta metodológica para a operacionalização do PDI”, aprovado no edital Pró-administraçã/CAPES.

\section{MEDIDA DE AVALIAÇÃO DA RESISTÊNCIA A MUDANÇA COM BASE NA TRI: DESENHO E OPERACIONALIZAÇÃO DE UM INSTRUMENTO PARA O MEES}

A Figura 3 apresenta a configuração do instrumento de medida proposto. O Mapa Estratégico da Educação Superior (MEES), fundamentado em um Sistema de Gestão Integrado, constitui-se em uma proposta metodológica para a operacionalização do PDI em IES.

$\mathrm{O}$ instrumento de medida para avaliar a resistência à mudança tem o propósito de identificar e minimizar as resistências no processo de consolidação do MEES. O plano de implementação do MEES se constitui das três fases descritas na seção quatro deste artigo. O desenho de um instrumento de medida para avaliar a resistência à mudança está alicerçada nos autores referenciados nas seções anteriores deste artigo.

Tem por base conceitual as seguintes abordagens: a gestão da mudança desdobrada em três itens: mudança (conceito, tipos e formas de implementação); Resistência (principais causas de resistência à mudança); e atitudes (positivas e negativas em relação à mudança). A Teoria psicométrica é utilizada com ferramenta para a elaboração do conjunto de itens.

A Teoria de Resposta ao Item (TRI), por meio do modelo de desdobramento graduado generalizado (GGUM), é utilizada para definir a escala de medida da resistência à mudança e com esta escala será possível estimar o grau de resistência à mudança nas três fases de implementação do MEES.

O instrumento de coleta de dados consiste em um questionário elaborado com base nos seguintes indicadores de resistência à mudança organizacional selecionados para esta modelagem: abertura à experiência, informação, participação, recompensa intrínseca, confiança na gerência, ceticismo em relação à mudança, medo ou receio em relação à mudança e resiliência psicológica, comprometimento organizacional, poder-prestigio, autoeficácia e rigidez cognitiva os quais foram identificados no referencial teórico (Kotter; Schlesinger, 1979; Robbins, 2002; Judson, 1966; Keneth, 1995; Taylor, 1988; Zander, 1950; Oreg, 2006; Wanberg \& Banas, 2000; Lawrence, 1978; Coch \& French, 1948; Judge et al. 1999) que vai ser desenvolvido na primeira etapa. 
A segunda etapa consiste na definição do tamanho da amostra representativa da comunidade acadêmica que responderá aos questionários. Como a TRI exige uma amostra de grande amplitude, e com o propósito de manter-se em sintonia com a metodologia de implementação do MEES será definida uma amostra representativa da comunidade acadêmica de IES por tipo (universidade, centro universitário e faculdade) e região (norte, nordeste, sul, sudeste e centro-oeste)A comunidade acadêmica é constituída pelos seguintes segmentos; gestores, professores, estudantes de graduação, estudantes de pós-graduação e funcionários técnico-administrativos.

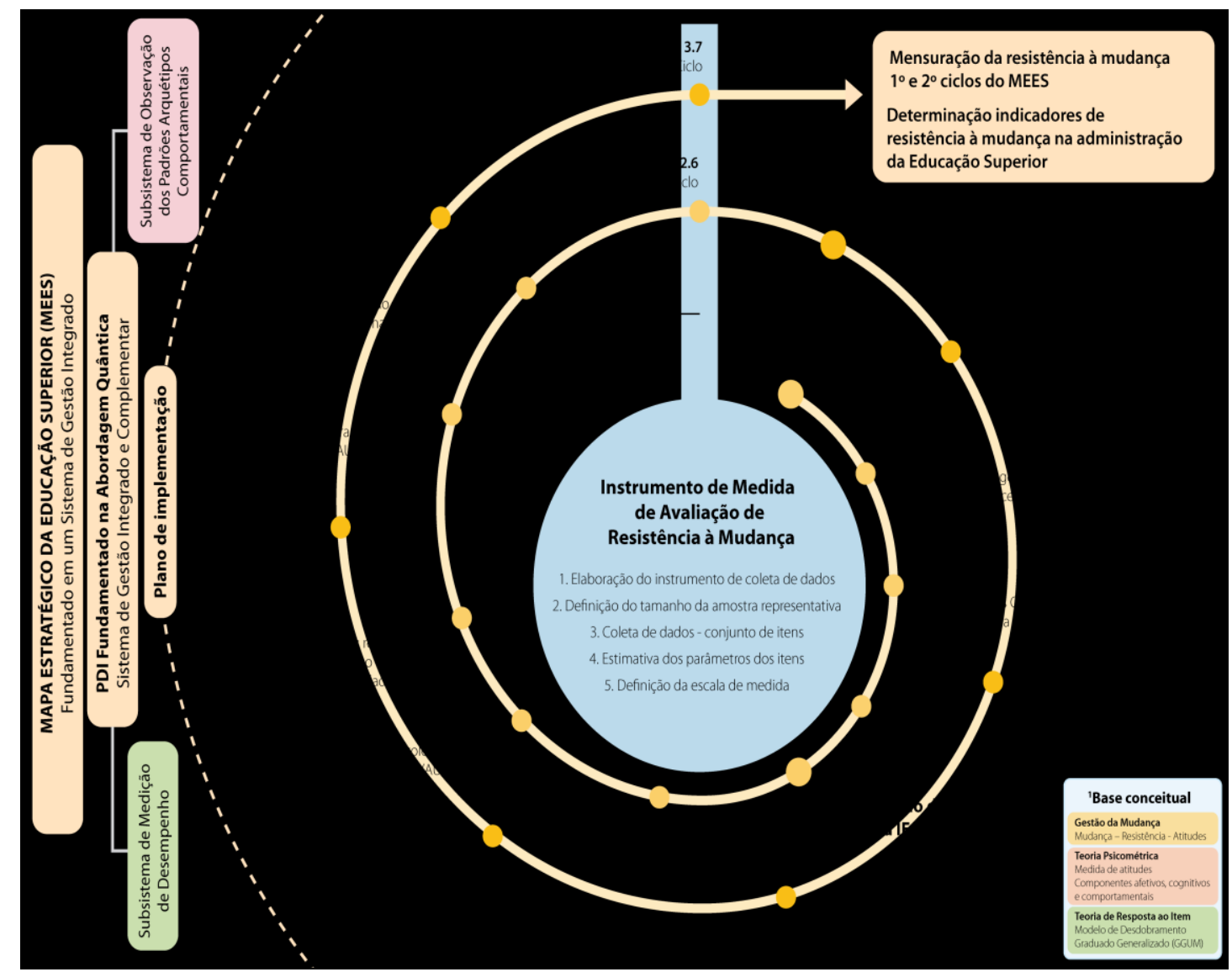

Figura 3 Modelagem de um Instrumento de Medida de Avaliação da Resistência a Mudança na Administração da Educação Superior: Desenho para o MEES

$\mathrm{Na}$ terceira etapa será efetuada a coleta de dados com base em um conjunto de itens definidos previamente a partir dos indicadores de resistência à mudança selecionados para esta modelagem.

Na quarta etapa será realizada a aplicação do modelo de desdobramento da TRI aos dados coletados. Nesta etapa vão ser estimados os parâmetros dos itens e dos respondentes por meio do software GGUM2004 desenvolvido por Roberts et al. (2004). 
$\mathrm{Na}$ quinta etapa é feita a definição da escala de medida com base nos parâmetros estimados dos itens. Como os itens e os respondentes estão na mesma unidade de medida, então é possível fazer comparações entre os diferentes comportamentos, bem como, saber onde cada respondente se localiza na escala e, constatar qual é o comportamento de cada respondente frente à mudança organizacional. É possível também identificar as causas que estão promovendo a resistência. Nos modelos de desdobramentos da TRI os itens são colocados de acordo com seu conteúdo e os respondentes são colocados de acordo com seu traço latente, ambos variando entre negativo, neutro a positivo (BORTOLOTTI, 2010).

A efetivação destas cinco etapas permitirá a mensuração da resistência à mudança nas três fases de implementação do MEES. Com este conhecimento a gerência pode desenvolver um planejamento adequado para a implantação da mudança de forma que procure minimizar qualquer resistência. As informações que serão obtidas pelo instrumento proporcionarão aos gestores de mudança conhecimento do fenômeno resistência à mudança e assim podem gerenciá-la de forma a não afetar o resultado da implementação, pelo contrário, transformar em benefício.

\section{CONSIDERAÇÕES FINAIS}

Em um mundo marcado por disparidade de rendas e doenças causadas pelo stress, alimentação e poluição a concepção da educação superior têm um papel fundamental. Apesar dos avanços, a gestão das organizações tem como características principais a competitividade na busca de espaços na sociedade. Ações individuais são preponderantes a formação de arranjos institucionais, parcerias, redes e clusters.

Sistemas, modelos, ferramentas e instrumentos de gestão que integram a dimensão humana, a colaboração e a co-evolução da educação superior aos seus planos, objetivos e metas são ainda incipientes. E, requerem uma mudança cultural, que gera como consequência uma resistência a qualquer alteração no status quo.

Bortolotti (2010), inova ao propor um instrumento de medida que visa avaliar a resistência a mudança nas organizações por meio da mensuração de dados intangiveis , com base em indicadores de atitudes que integram componentes afetivos, cognitivos e comportamentais. Corrêa $(2007,2008)$, também inova ao propor um sistema de gestão integrado cuja representação visual é o mapa estratégico da educação superior (MEES). 
O Instrumento de medida de avaliação da resistência na administração da educação superior tem o propósito de contribuir para a eficácia na implementação do MEES. Visa também identificar os principais indicadores de resistência a mudança na administração da educação superior.

\section{REFERÊNCIAS}

ALPERSTEDT, G. D. Adaptação estratégica em organização universitária: um estudo qualitativo na Universidade do Sul de Santa Catarina. 2000. Tese (Engenharia de Produção). Programa de Pós-Graduação em Engenharia de Produção, Centro Tecnológico, Universidade Federal de Santa Catarina, Florianópolis, 2000.

ANDRADE, D. F., TAVARES, H. R., VALLE, R. C. Teoria de Resposta ao Item: conceitos e aplicações. ABE - Associação Brasileira de Estatística, 4º SINAPE, 2000.

ARAUJO, E. A. C. de. ; ANDRADE, D. F.; BORTOLOTTI, S. L. V. Teoria da Resposta ao item. Revista da Escola de Enfermagem , USP, 43 (Esp):1000-8, 2009.

ARMENAKIS, A. A., HARRIS, S. G., MOSSHOLDER, K. W. Creating readiness for organizational change. Human Relations, 46, 681-703, 1993.

BARTUNEK, J. M.; MOCH, M. K. First-order, second-order, and third-order change and organization development interventions: A cognitive approach. Journal of Applied

Behavioral Science, v. 23, p. 483-500, 1987.

BEMMELS B.; RESHEF, Y. Manufacturing employees and technological change. Journal of Labour Research, v. 12, n. 3, Summer, p. 231-46, 1991.

BLOCK, P. -Flawless consulting\|, In McLennan, R., Managing Organizational Change, Prentice Hall, Englewood Cliffs, NJ, 1989.

BORTOLOTTI, S. L. V. Resistência à mudança organizacional: Medida de avaliação por meio da teoria da resposta ao item (Tese de doutorado). Programa de Pós Graduação em Engenharia de Produção da Universidade Federal de Santa Catarina, 2010.

BOVEY, W. H., HEDE, A. Resistance to organizational change: the role of defence mechanism. Journal of Managerial Psychology, v. 16, n.7, p. 534-548, 2001.

BRASIL. Lei n ${ }^{\circ} 10.861$, de 14 de abril de 2004. Institui o Sistema Nacional de Avaliação da Educação Superior - SINAES - e dá outras Providências. Diário Oficial da União, Brasília, DF, DOU n 072 , de 15/4/2004, seção 1, p. 3-4.

COCH, L., FRENCH, J. R. P., Jr. Overcoming resistance to change. Human Relations, v.1, $512-532,1948$. 
COGHLAN, D. A person-centred approach to dealing with resistance to change. Leadership \& Organization Development Journal, vol. 14, 4; ABI/INFORM Global, pg. 10-14, 1993.

CONNER, D. R. Gerenciando na velocidade da mudança: como gerentes resilientes são bem sucedidos e prosperam onde outros fracassam. Tradução Andréia Alves, Rio de Janeiro: Infobook, 1995.

COOMBS, C. H. A theory of data. New York: Wiley, 1964.

CORRÊA, A. C. A Divulgação da Produção Científica Como um Ítem de Controle de Qualidade da Pesquisa, 1998. Dissertação (Mestrado em Engenharia de Produção). Programa de Pós-Graduação em Engenharia de Produção, Centro de Tecnologia, Universidade Federal de Santa Maria, Santa Maria, 1998.

Sistema de Gestão para as IES com base no Balanced Scorecard como um Sistema Complexo Adaptativo: Uma proposta metodológica para a operacionalização do PDI. In: VII Colóquio de Gestión Universitaria de América Del Sur, 2007, Mar Del Plata. Argentina. Anais... Mar Del Plata, 2007, 1a.

Um olhar reflexivo à dimensão políticas de pessoal das IFES por meio do Programa de Auto-avaliação Institucional da UFSC. In: VII Colóquio de Gestión Universitaria de América Del Sur, 2007, Mar Del Plata. Anais... Mar Del Plata, 2007, 1 b.

O Balanced Scorecard como um Sistema Adaptativo Complexo: uma abordagem quântica à estratégia. 2005. 360 f. Tese (Engenharia de Produção). Programa de PósGraduação em Engenharia de Produção, Centro Tecnológico, Universidade Federal de Santa Catarina, Florianópolis, 2005, $1^{\text {a }}$ Disponível em: http://teses.eps.ufsc.br/defesa/pdf/8758.pdf.

CORREA, A., COELHO, C. S. R., SELIG, P. M., Mapas Estratégicos do Balanced Scorecard como um SCA: Uma Abordagem Quântica à Estratégia. In: Encontro Nacional de Engenharia de Produção, 2005, Porto Alegre. Anais... XXV Encontro Nacional de Engenharia de Produção. Porto Alegre: Álvaro Gehlen de Leão, PUCRS, 2005, 1 b.

CORREA, A. C., ERDMANN, R. H., MELO, P. A. de., RISSI, M., TECCHIO, E. L. Mapa Estratégico para as IES fundamentado em um Sistema de Gestão Integrado: uma proposta metodológica para a implementação do PDI. In: VIII Colóquio Internacional sobre Gestión Universitaria en América del Sur, 2008, Assunción. Paraguay. Anais... Universidade Tecnológica Intercontinental, 2008. p. 1-16.

DEL VAL, M. P. Resistance to change a literature review and empirical study, Management Decision, Vol. 41 Nos 1/2, pp. 148-55, 2003.

FREIRE, P., SHOR, I. Medo e ousadia Rio de Janeiro. Paz e Terra, 1986.

GANDIN, D., GANDIN, L. A. Temas para um projeto político-pedagógico. Vozes Ltda, $5^{\text {a }}$ ed., Petrópolis, RJ, 1999. 
GIANGRECO, Antonio. Conceptualisation and Operationalisation of Resistance to Change. Liuc Papers n. 103, Série Econômica aziendale11, Suppl. Marzo, 2002.

GEISLER, D. Bottom-feeders: people who reject changel, Executive Excellence, 18 (12) 19, 2001 .

GILMORE, T.N., Shea, G.P. and Unseem, M. - Side effects of corporate cultural transformations, Journal of Applied Behavioral Science, Vol. 33, pp. 174-89, 1997.

GORESKE, A. Mudança organizacional - Capacidade de adaptação. Revista do Granbery $1 \backslash 272$ - Semestre 2, 2007.

HACKMAN J.; OLDHAM, G. R. Motivation through the design of work: Test of a theory. Organizational Behavior and Human Performance, 16(2), 250 - 279, 1980.

INEP - Instituto Nacional de Estudos e Pesquisas Educacionais Anísio Teixeira. Sistema Nacional da Avaliação da Educação Superior: da concepção à regulamentação. $5^{\mathrm{a}} \mathrm{ed}$. Ampliada. Brasília, 2009.

JUDSON, A. S. Relações humanas e mudanças organizacionais. São Paulo: Editora Atlas, 1980.

JUDGE, T. A., THORESEN, C. J., PUCIK, V., WELBOURNE, T. M. Managerial coping with organizational change: A dispositional perspective. Journal of Applied Psychology, v. 84 , n. 1, p. $107-122,1999$.

KENETH, H. Scaling the wall of resistance. Training \& Development, v. 49, n.10, p-15-18, 1995.

KOTTER, J. P. Leading change: why transformation efforts fail. Harvard Business Review, Boston, v. 73, n. 2, p. 59-67, Mar.1995.

KOTTER, J. Oito erros fatais\|. In: JÚLIO, C.;Neto, J. (Org.), Inovação e mudança: autores e conceitos imprescindíveis, São Paulo:Publifolha, p. 93-100, 2001.

KOTTER, J. P., \& SCHLESINGER, L. A. Choosing strategies for change. Harvard Business Review, Boston, v. 57, n. 2, p. 106-113, Mar/Apr 1979.

LAWRENCE, P. R. How to deal with resistance to change. Harvard Business Review, 32, no. $3,49-57,1978$.

LAU C. M.; WOODMAN R. W. Understanding organizational change: A schematic perspective. Academy of Management Journal, 38(2), 537 - 554, 1995.

LIMA, S. M. V. Mudança organizacional: teoria e gestão. 1a ed. Rio de Janeiro: Editora FGV, 2003.

MARTIN, H. H. How we Shall Overcome Resistance. Training and Development Journal, v. 29, n. 10, 32, Madison: OCT, 1975. 
MAURER, R. Using Resistance to build support for Change. The Journal for Quality and Participation, 19 (3), P. 56-66, 1996A 1997.

Transforming Resistance. HR Focus, v. 74, n. 10, p. 9-10; ABI/INFORM Global, oct

MENDES, J. A Resistência às Tecnologias de Informação, Causas, Efeitos e Estratégias de Superação: Estudo de Caso da Implementação do SAP R/3. 2001. Dissertação.

Universidade do Minho, Braga, 2001.

MEYER JR.; V. Gestão para a Qualidade e Qualidade na Gestão: O caso das Universidades. In: Seminário Internacional de Gestão pela Qualidade em Universidades, Anais...UDESC, Florianópolis, SC, 1997.

MINISTÉRIO DA EDUCAÇÃO - MEC. Avaliação Externa das Instituições de Educação Superior: Diretrizes e Instrumento. CONAES/INEP. Brasília, 2006.

MINK, O.G. eating new organizational paradigms for changel, International Journal of Quality \& Reliability Management, Vol. 9 No. 3, pp. 21-35, 1992.

MORGAN, G. Imagens da Organização. São Paulo: Atlas, 1996.

MORIN, E. A ciência com consciência. São Paulo: Petrópolis, 1998.

NADLER, D. A.; HACKMAN, Jr.; LAWLER, E. Comportamento Organizacional. Editora Campus, Rio de Janeiro, 1983.

NEIVA, E. R. Percepção de Mudança organizacional: o papel das atitudes e das características organizacionais. Tese de Doutorado, Universidade de Brasília, DF, Brasil, 2004.

NIKOLAOU, I., TOMPROU, M. AND VAKOLAR, M. Individuals' inducements and the role of personality: implications for psychological contracts. Journal of Managerial Psychology, Vol. 22 No. 7, pp. 649-63, 2007.

OREG, S. Personality, context and resistance to organizational change. European Journal of Work and Organizational Psychology, 15, 73-101, 2006.

O ${ }^{\text {ee }}$ CONNOR, C. A. Resistance: the repercussions of change. Leadership \& Organization Development Journal, v. 14, n. 6, 1993.

PASQUALI, L. Teoria e Métodos de Medida em Ciências do Comportamento. Brasília, INEP, 1996. 2003.

Psicometria: teoria dos testes na psicologia e na educação. Petrópolis, RJ: Vozes, 
Vozes, 2009.

Psicometria: Teoria dos testes na Psicologia e na Educação. 3. ed. Petrópolis, RJ:

PETTIGREW, A. É a cultura Organizacional administrável? In: FLEURY, M. T. et alli. Cultura e Poder nas Organizações. São Paulo: Atlas, 1989.

PEREIRA, M. J. B. Pressupostos da mudança. In: Na cova dos leões - o consultor como facilitador do processo decisório empresarial. São Paulo: Makron Books, Cap.1, p 3-12, 1999.

PIDERIT, S. K. Rethinking resistance and recognizing ambivalence: A multidimensional view of attitudes toward an organizational change. Academy of Management Review, 25(4), $783-794,2000$.

PORRAS, J.I. \& ROBERTSON, P.J. Organizational development: theory, practice, research. Handbook of Organizational Psychology, ed. MD Dunnette, LM HOUGH, 3, 719-822. Palo Alto, C.A: Consultant Psychology Press. 2a edição, 1992.

ROBERTS, J. S.; DONOGHUE, J. R.; LAUGHLIN, J. E. A general item response theory model for unfolding unidimensional polytomous responses. Applied Psychological Measurement, 24, 3-32, 2000.

ROBERTS, J. S.; FANG, H.; CUI, W.; WANG, Y. GGUM. A Windows-based program to estimate parameters of the generalized graded unfolding model. Manuscript in preparation, 2004.

ROBBINS, S. P. Comportamento organizacional. São Paulo: Pretice Hall, 2002.

RONCA, A. C. C., FREITAS, A. de A, CRAVIERO, C. B. A., Cordão, F. A., Trindad, H. H. C., Speller, P., Indicações para subsidiar a construção do Plano Nacional de Educação 2011-2020. Ministério da Educação, agosto de 2009. Recuperado de:

http://portal.mec.gov.br/dmdocuments/pne 200809.pdf.

SANTOS, A. Didática sob a ótica do pensamento complexo. Sulina, Porto Alegre, 2003.

SHEIN, E. H. Psicologia Organizacional. $3^{\text {a }}$ Edição, Rio de Janeiro: Prentice-Hall do Brasil, 1982.

SELF, D. R., SCHRAEDER, M. Enhancing the success of organizational change. Matching readiness strategies with sources of resistance., Leadership \& Organization Development Journal. 30(2):167-182, 2009.

SPIKER, B. K.; LESSER, E. We have met the enemy. Journal of Business Strategy, v. 16, n. 2, p. 17-21, 1995.

TAYLOR, R. E. Reducing Resistance to New Marketing Strategies. Business Forum, vol. 13, n. $2,1988$. 
TRIGUEIRO, M. G. S. Indicadores de Qualidade na Universidade: um desafio para a Avaliação Institucional. Universidade: a busca da qualidade, São Paulo, v. 1, n. 6, p. 320330, novembro/dezembro, 1994.

VALA, J.; MONTEIRO, M. B.. Psicologia social. 7. ed Lisboa: Fundação Calouste Gulbenkian, 2006.

VAN DER LINDEN, W. J.; HAMBLETON, R. K. Handbook of Modern Item Response Theory. New York: Spring- Verlag, 1997.

VEIGA, I. P. A. (Org.) et al. Projeto Político-pedagógico da escola: uma construção possível. $3^{\mathrm{a}}$ ed. Campinas, SP: Papirus, 1997.

WANBERG, C. R.; BANAS, J. T. Predictors and outcomes of openness to changes in a reorganizing workplace. Journal of Applied Psychology, 85(1), 132 - 142, 2000.

WADELL, D.; SOHAL, A. Resistance: a constructive tool for change management. Management Decision, v. 36 n. 8, p. 543-8, 1998.

WOOD JR (Coord.) Mudança Organizacional. 4 ed. São Paulo: Atlas, 2004.

WEICK, K.E. \& QUINN, R.E. Organizational change and development. Annual Review of Psychology, 50, 361-387, 1999.

ZANDER, A. Resistência às modificações: análise e prevenção. In: BALCÃO, Y. F.; CORDEIRO, L. L. 3.ed. O comportamento humano na empresa. Rio de Janeiro: Fundação Getúlio Vargas, p. 371-80, 1977.

ZALTMAN, G.; DUNCAN, R.. Strategies for planned change. New York: Wiley, 1977. 